\title{
ADDITIONS TO SYMPLOCACEAE OF THE OLD WORLD INCLUDING NEW CALEDONIA
}

\author{
H.P. NOOTEBOOM \\ Nationaal Herbarium Nederland, Universiteit Leiden branch, \\ P.O. Box 9514, 2300 RA Leiden, The Netherlands \\ e-mail: Nooteboom@nhn.leidenuniv.nl
}

\begin{abstract}
SUMMARY
Some name changes and new names are published belonging to a new revision of Symplocaceae of the Old World. Several species described after 1975 were reduced.
\end{abstract}

Key words: Symplocos, name changes, reinstated taxa, reduced taxa.

\section{INTRODUCTION}

The author published in 1975 'Revision of the Symplocaceae of the Old World, New Caledonia excepted'. Because of the many variable taxa the dichotomous keys appeared difficult to use. Therefore the author made all the descriptions again with the DELTA package for Windows. The database was used to make an interactive key to be used with INTKEY. New insights as well as the use of DELTA made it possible to reinstate some taxa. Some other taxa, published after 1975 by other authors, are accepted, others are reduced. The name changes en reductions are published here.

In the new treatment 237 taxa are recognised. The entire revision including the illustrated interactive key and a complete list of accepted taxa and synonyms is published on a CD-ROM which goes with this article, as well as on the website of the Nationaal Herbarium Nederland, Universiteit Leiden branch.

Most species are now relatively easy to identify (nearly all taxa are illustrated), with exception of many New Guinean taxa. The complicated geological and biological history of New Guinea is responsible for incomplete speciation in many groups in New Guinea, e.g. in Symplocos. A study of several years using DNA and morphology is needed to clarify the New Guinean taxa.

\section{Symplocos anomala Brand var. morrisonicola (Hayata) S. S. Ying}

Symplocos anomala Brand var. morrisonicola (Hayata) S. S. Ying (1987) 593. - Symplocos morrisonicola Hayata (1908) 190, pl. 27; R.F. Wu (1987) 15, pl. 46, photo 78, 79; Nagam. (1998) 119. - Bobua morrisonicola (Hayata) Kaneh. \& Sasaki (1928) 331. - Symplocos anomala Brand (1901) 67, p.p.; Noot. (1975) 126, p.p.; R.F. Wu \& Noot. (1996) 242, p.p. - Syntypes: S. Nagasawa 737, Suizan, Oct. 1905; G. Nakahara, Tozan, Oct. 1906; and T. Kawakami \& U. Mori 1702, Mt Morrison, Oct. 1906, all in Taiwan (n.v.). 
Symplocos doii Hayata (1915) 96, f. 25h. - Bobua doi (Hayata) Kaneh. \& Sasaki (1928) 330. - Symplocos cuspidata Brand var. doii (Hayata) S.S. Ying (1975) 565. - Type: T. Doi 1912 (n.v.), Rinpiho, Taiwan.

Symplocos kiraishiensis Hayata (1920) 68, pl. 4. - Symplocos morrisonicola Hayata var. kiraishiensis (Hayata) T. Mori (1934a) 195; (1934b) 240. - Symplocos anomala Brand var. morrisonicola (Hayata) S.S. Ying forma kiraishiensis (Hayata) S. S. Ying (1987) 595. - Type: Y. Shimada, Kiraishi, Taiwan, March 1918.

Bobua morrisonicola (Hayata) Kaneh. \& Sasaki var. matudai Hatus. (1935) 211. - Symplocos anomala Brand var. morrisonicola (Hayata) S.S. Ying forma matudai (Hatus.) S.S. Ying (1987) 595. - Symplocos morrisonicola Hayata var. matudai (Hatus.) S. S. Ying (1975) 567. - Type: H. Matuda (n.v.), Mt Buisan, Taiwan, 31 Dec. 1918.

\section{Symplocos congesta Benth.}

Symplocos congesta Benth. (1861) 211; Nagam. (1998) 108. - Symplocos glomerata King ex Clarke var. congesta (Benth.) Noot. (1975) 202; Noot. \& Vidal (1977) 40; R.F. Wu \& Noot. (1996) 251. - Type: Champion 413 (K), Hongkong.

\section{Symplocos cordifolia Thwaites}

Symplocos cordifolia Thwaites (1860) 187. - Lodhra cordifolia Miers (1879) 301. - Symplocos macrophylla Wall. ex DC. var. cordifolia (Thwaites) Noot. (1975) 233. - Type: Thwaites CP 271 (K; iso BM, CGE, FI, LE, MEL, P), Ceylon.

\section{Symplocos doormannensis Brand}

Symplocos doormannensis Brand (1924) 188. - Symplocos cochinchinensis Brand var. doormannensis (Brand) Noot. (1975) 168. - Lectotype: H.J. Lam 1985 (L; iso BO), Doormantop, New Guinea.

\section{Symplocos heishanensis Hayata}

Symplocos heishanensis Hayata (1915) 101, t. 28; T. Mori (1934b) 231; Kaneh. (1936) t. 545; Noot. (1975, sub S. sumuntia Buch.-Ham. ex D. Don); R.F. Wu \& Noot. (1996) 245. - Bobua heishanensis Kaneh. \& Sasaki (1928) 331. - Syntypes: T. Ito \& B. Hayata s.n. (TOFO n.v.), Taiwan, and R. Kanehira \& I. Tanaka s.n. (n.v.), Taiwan, Nimandaira, April 1914.

Symplocos eriostroma Hayata (1915) 99, f. 25c; T. Mori (1934b) 228; Kaneh. (1936) 585; Noot. (1975, sub S. modesta Brand); Nagam. (1998) 111. - Type: S. Sasaki, no 2. (n.v.), Taiwan, Mt Arisan, Jan. 1911.

Symplocos macrostroma Hayata (1915) 107, t. 25, 5; T. Mori (1934b) 237; Noot. (1975: 295, sub dubious). - Symplocos japonica Hayata (1911) 188, non DC (1844). - Palura macrostroma (Hayata) Kaneh. \& Sasaki (1928) 332. - Bobua macrostroma (Hayata) Kaneh. \& Sasaki (1930) 409. - Type: Mori 2658 (n.v.), Taiwan, Kappan-zan.

Symplocos risekiensis Hayata (1915) 112, t. 35; Noot. (1975, sub S. oreades). - Bobua risekiensis Kaneh. \& Sasaki (1928) 332. - Type: T. Kawakami \& U. Mori 3551 (n.v.), Taiwan, Nanto, Risekisan.

Symplocos oreades Guillaumin (1932) 174, excl. syn. S. poilanei; (1933) 1032; Noot. (1975) 256. - Type: Poilane 17046 (P; iso NY), Tonkin, Col de Lo qui ho, near Chapa.

Symplocos pittosporifolia Hand.-Mazz. (1943) 25; Noot. (1975, sub S. oreads). - Type: Ching 7843 (W; iso NY). 


\section{Symplocos obtusa Wall. ex G. Don var. cucullata Thwaites}

Symplocos obtusa Wall. ex G. Don var. cucullata Thwaites (1860) 185. - Symplocos cucullata Brand (1901) 56 (in: Noot. (1975) 243 under var. obtusa); Noot. (1981) 466. - Type: Thwaites CP 2835 (K; iso BM, LE, P), Ceylon.

\section{Symplocos paniculata (Thunb.) Miq.}

Symplocos paniculata (Thunb.) Miq. (1865) 102, Noot. (1975) 258; R.F. Wu \& Noot. (1996) 252.

- Type: Thunberg s.n. (UPS n.v.), Japan.

Symplocos chinensis (Lour.) Druce (1917) 650; Nagam. (1998) 105. - Type: Loureiro s.n. (P). Symplocos coreana (Lév.) Ohwi (1953) 83; Nagam. (1993) 195. - Type: Taquet 1106 (E), Korea. Symplocos sawafutagi Nagam. (1993) 199, pl. 53d. - Type: Anonymous s.n. (TI n.v.).

Symplocos tanakana Nakai (1918) 227; Nagam. (1993) 206, pl. 55b. — Lectotype (Nagamasu, 1993): Nakai 6411 (TI), Quelpart, Korea.

Note - Nagamasu (1993: 195) recognises several species, forming together his section Palura. I failed, when trying to identify some collections, already in the first lead. The leaves are definitely not glaucous on the undersurface in what is identified by Nagamasu as Symplocos tanakana. That, and the bark being transversely fissured (a character I could not observe) are the only differences between S. tanakana and the rest of his species. The differences between his $S$. coreana and $S$. sawafutagi are, i.e.: Leaves ovate to obovate versus leaves obovate to elliptic. In my opinion the latter is part of the first. And: apex acuminate to caudate versus abruptly acuminate to acuminate. According to me this is a continuous variation. Part of the variability in $S$. paniculata probably is due to different ecology, therefore populations may differ from each other because they have a different ecology. So his $S$. coreana occurs often on ridges or on mountain slopes and $S$. sawafutagi is found in deciduous forest, usually on alluvium in shaded places along streamlets. The different habitat being responsible for the differences, they should be designated as ecotypes, not species.

\section{Symplocos pendula Wight var. hirtistylis (Clarke) Noot.}

Symplocos pendula Wight var. hirtistylis (Clarke) Noot. (1975) 42; Noot. \& J.E. Vidal (1977) 15; R.F. Wu \& Noot. (1996) 252. - Type: Maingay 2580 (K), Malaya.

Symplocos sonoharai Koidz. (1928) 6; Nagam. (1993) 192; (1998) 123. - Lectotype (Nagamasu, 1993): S. Sonohara 1 (KYO), Utchina Is., Ryukyu Isls.

Symplocos yanchunensis H.G. Ye \& J. Xing (2003) 277. - Type: H.G. Ye 6546 (IBSC), China, Guangdong, Yangchun, Hewei Mountain.

9. Symplocos ramosissima Wall. ex G. Don var. xylopyrena (C. Y. Wu ex Y.F. Wu) Noot, comb. nov.

Symplocos xylopyrena C.Y. Wu ex Y.F. Wu (1982) 91; R.F. Wu \& Noot. (1996) 246. - Type: Qinzhang Exped. 57-1814 (IBSC), Xizang, Mêdog xian.

10. Symplocos sumuntia Buch.-Ham. ex D. Don var. modesta (Brand) Noot., var. nov. \& stat nov.

Symplocos modesta Brand (1901) 66; Noot. (1975) 239; R.F. Wu \& Noot. (1996) 246; Nagam. (1998) 117. - Type: S. Sasaki, no. 2 (n.v.), Formosa, Jan. 1911. 


\section{REFERENCES}

Bentham, G. 1861. Flora Hongkongensis: 211. Reeve, London.

Brand, A. 1901. In: H.G.A. Engler, Das Pflanzenreich 6, IV. Engelmann, Berlin.

Brand, A. 1924. Symplocaceae. Nova Guinea 14: 186-189.

De Candolle, A.P. 1844. Prodromus Systemati Naturalis Regni Vegetabilis 8: 257. Treutel \& Wurtz, Paris, London, Strasbourg.

Druce, G.C. 1917. Symplocaceae. Bot. Soc. Exch. Club Brit. Isles 4: 650.

Guillaumin, J. A. 1932. Espèces et localités nouvelles de Symplocos. Bull. Soc. Bot. France 79: 174.

Guillaumin, J.A. 1933. In: P.H. Lecomte, Fl. Indo-Chine 3: 992-1034.

Handel-Mazzetti, H. 1943. Symplocaceae aus China. Beih. Bot. Centralbl. 62-B: 25.

Hatusima, S. 1935. Contributiones ad Dendrologiam Nipponiae Australis. Acta Phytotax. Geobot. 4: 211 .

Hayata, B. 1908. Flora Montana Formosana. J. Coll. Sci. Imp. Univ. Tokyo 25: 190, pl. 27.

Hayata, B. 1911. Materials for a Flora of Formosa. J. Coll. Sci. Imp. Univ. Tokyo 30: 188.

Hayata, B. 1915. Icones PIantarum Formosanarum 5: 96, 101, 107, 112. Government of Formosa, Taihoku.

Hayata, B. 1920. Icones PIantarum Formosanarum 9: 68, 96. Government of Formosa, Taihoku.

Kanehira, R. 1936. Formosan Trees. Government of Formosa, Taihoku.

Kanehira, R \& S. Sasaki. 1928. List of plants of Formosa. Natural History Society of Formosa, Taihoku.

Kanehira, R \& S. Sasaki. 1930. A catalogue of the Government Herbarium. Department of Forestry, Taihoku.

Koidzumi, G. 1928. Plantae Novae Amami-Oshima.

Miers, J. 1879. On the Symplocaceae. J. Linn. Soc., Bot. 17: 301.

Miquel, F.A.W. 1865. Prolusio Florae Japonicae. Ann. Mus. Bot. Lugduno-Batavi 3: 102.

Mori, T. 1934a. Materials to the Arboreal Flora of Formosa. Trans. Nat. Hist. Soc. Formosa 24: 195.

Mori, T. 1934b. The Symplocaceae of Formosa. Sylvia 5: 231, 237, 240.

Nagamasu, H. 1993. The Symplocaceae of Japan. Contr. Biol. Lab. Kyoto University.

Nagamasu, H. 1998. Flora of Taiwan 2nd ed., 4: 101-127. Taipei, Taiwan, ROC.

Nakai, T. 1918. Notulae ad Plantas Japonicae et Koreae XVIII. Bot. Mag. (Tokyo) 32: 227.

Nooteboom, H.P. 1975. Revision of the Symplocaceae of the Old World. Universitaire Pers Leiden.

Nooteboom, H.P. 1981. A revised handbook to the Flora of Ceylon 3: 454-478. Amerind Publishing Co., New Delhi.

Nooteboom, H.P. \& J.E. Vidal. 1977. Flore du Cambodge, de Laos et du Viêtnam 16. Muséum d'Histoire Naturelle, Laboratoire de Phanerogamie, Paris.

Ohwi, J. 1953. New names and new combinations adopted in my 'Flora of Japan'. Bull. Natl. Sci. Mus., Tokyo 33: 83.

Thwaites, G.H.K. 1860. Enumeratio Plantarum Zeylaniae 3: 185, 187. Dulau \& Co., London.

Wu, R.F. 1987. Symploceae. In: Fl. Reipubl. Popularis Sin. 60: 1-77.

Wu, R.F. \& H.P. Nooteboom. 1996. Symplocaceae. Flora of China 15: 235-252. Science Press (Beijing) \& Missouri Botanical Garden (St. Louis).

Wu, Y.F. 1982. Symplocaceae novae e Flora Tibetica. Acta Phytotax. Sin. 20: 91.

Ye, H.G. \& J. Xing. 2003. A new species and a new synonym of Symplocos (Symplocaceae) from China. J. Trop. Subtrop. Bot. 11: 277.

Ying, S.S. 1975. Symplocos. Bull. Exp. Forest Natl. Taiwan Univ. 116: 565, 567.

Ying, S.S. 1987. Colour. Illustr. Fl. Taiwan 2: 593. 


\section{INDEX}

The numbers refer to the species numbers used. The accepted names are in roman type, new names in bold and synonyms in italics.

\author{
Bobu(a) doi (Hayata) Kaneh. \& Sasaki 1 \\ heishanensis Kaneh. \& Sasaki 5 \\ macrostroma (Hayata) Kaneh. \& Sasaki 5 \\ morrisonicola (Hayata) Kaneh. \& Sasaki 1 \\ var. matudai Hatus. 1 \\ risekiensis Kaneh. \& Sasaki 5 \\ Lodhra cordifolia Miers 3 \\ Palura macrostroma (Hayata) Kaneh. \& \\ Sasaki 5 \\ Symplocos Desf. \\ anomala Brand p.p. 1 \\ var. morrisonicola (Hayata) S.S. Ying 1 \\ forma kiraishiensis (Hayata) S.S. Ying 1 \\ forma matudai (Hatus.) S.S. Ying 1 \\ chinensis (Lour.) Druce 7 \\ cochinchinensis Brand \\ var. doormannensis (Brand) Noot. 4 \\ congesta Benth. 2 \\ cordifolia Twaites 3 \\ coreana (Lév.) Ohwi 7 \\ cucullata Brand 6 \\ cuspidata Brand \\ var. doii (Hayata) S.S. Ying 1 \\ doii Hayata 1 \\ doormannensis Brand 4 \\ eriostroma Hayata 5 \\ glomerata King ex Clarke \\ var. congesta (Benth.) Noot. 2 \\ heishanensis Hayata 5
}

\author{
(Symplocos) \\ japonica Hayata 5 \\ kiraishiensis Hayata 1 \\ macrophylla Wall. ex DC. \\ var. cordifolia (Thwaites) Noot. 3 \\ macrostroma Hayata 5 \\ modesta Brand 10 \\ morrisonicola Hayata 1 \\ var. kiraishiensis (Hayata) T. Mori 1 \\ var. matudai (Hatus.) S.S. Ying 1 \\ obtusa Wall. ex G. Don \\ var. cucullata Thwaites 6 \\ oreades Guillaumin 5 \\ paniculata (Thunb.) Miq. 7 \\ pendula Wight \\ var. hirtistylis (Clarke) Noot. 8 \\ pittosporifolia Hand.-Mazz. 5 \\ ramosissima Wall. ex G. Don \\ var. xylopyrena (C.Y. Wu ex Y.F. Wu) \\ Noot. 9 \\ risekiensis Hayata 5 \\ sawafutagi Nagam. 7 \\ sonoharai Koidz. 8 \\ sumuntia Buch.-Ham. ex D. Don \\ var. modesta (Brand) Noot. 10 \\ tanakana Nakai 7 \\ xylopyrena C.Y. Wu ex Y.F. Wu 9 \\ yanchunensis H.G. Ye \& J. Xing 8
}

